TRANSACTIONS OF THE

AMERICAN MATHEMATICAL SOCIETY

Volume 364, Number 12, December 2012, Pages 6315-6333

S 0002-9947(2012)05500-0

Article electronically published on June 29, 2012

\title{
RATIONALITY OF RATIONALLY CONNECTED THREEFOLDS ADMITTING NON-ISOMORPHIC ENDOMORPHISMS
}

\author{
DE-QI ZHANG
}

\begin{abstract}
We prove a structure theorem for non-isomorphic endomorphisms of weak $\mathbb{Q}$-Fano threefolds or, more generally, for threefolds with a big anticanonical divisor. Also provided is a criterion for a fibred rationally connected threefold to be rational. As a consequence, we show (without using the classification) that every smooth Fano threefold having a non-isomorphic surjective endomorphism is rational.
\end{abstract}

\section{INTRODUCTION}

We work over the field $\mathbb{C}$ of complex numbers. We shall prove Theorems 1.1, 1.2 and 1.3

Theorem 1.1. Let $X$ be a $\mathbb{Q}$-factorial projective threefold having only terminal singularities, a surjective endomorphism $f: X \rightarrow X$ of degree $>1$, and a $K_{X^{-}}$ negative extremal contraction $X \rightarrow Y$ with $\operatorname{dim} Y \in\{1,2\}$. Suppose either one of the following three conditions:

(1) $X$ is Gorenstein.

(2) $K_{X}^{3} \neq 0$.

(3) The ramification divisor $R_{f}$ is non-zero.

Suppose further that $X$ is rationally connected. Then $X$ is rational.

Theorem 1.2. Let $X$ be a smooth Fano threefold with a surjective endomorphism $f: X \rightarrow X$ of degree $>1$. Then $X$ is rational.

Here is a sketch of the proof of Theorem [1.2 using Theorem 1.1. Let $X \rightarrow X_{1}$ be the composite of blowdowns between smooth threefolds such that $X_{1}$ is a primitive smooth Fano threefold in the sense of [15]. The morphism $f$, replaced by some power, descends to a surjective morphism $f_{1}: X_{1} \rightarrow X_{1}$, using the finiteness of extremal rays in the Mori cone $\overline{\mathrm{NE}}(X)$. If $\rho\left(X_{1}\right)=1$, then $X_{1} \cong \mathbb{P}^{3}$ by [1] or [6], and we are done! Suppose that $\rho\left(X_{1}\right) \geq 2$. By [15, Theorem 5], $X_{1}$ has an extremal contraction of conic bundle type, so $X_{1}$ is rational by Theorem 1.1. Alternatively, as a referee remarked, such an $X_{1}$ fits one of the four families (or else is known to be rational; cf. [15]) whose explicit description may be used to give another probably simpler proof of Theorem 1.2

In [11, Kollár-Xu constructed many examples of endomorphisms $f: X \rightarrow X$ of degree $>1$ on quotients $X$ of projective spaces, which are Fano varieties of Picard

Received by the editors September 18, 2009 and, in revised form, September 25, 2010.

2010 Mathematics Subject Classification. Primary 14E20, 14J45, 14E08, 32H50.

Key words and phrases. Endomorphism, rationally connected variety, rationality of variety.

(C)2012 American Mathematical Society

Reverts to public domain 28 years from publication 6315 
number one with at worst terminal singularities; some $X$ might be irrational by invoking David Saltman's famous counterexamples to Noether's problem.

As another type of example, one takes an elliptic curve $E$ of period $\sqrt{-1}$ and the abelian variety $A=E^{n}$ with $n \geq 1$. Let $\mu_{4} \cong\langle\sqrt{-1}\rangle$ act diagonally on $A$. Then $X=A / \mu_{4}$ has only $\log$ terminal singularities and is rationally connected when $n \leq 3$. For $m \neq 0, \pm 1$, the multiplication map $m_{A}: A \rightarrow A(a \mapsto m a)$ descends to an endomorphism $f: X \rightarrow X$ of degree $m^{2 n}>1$. When $n=3$, it is not known whether $X$ is rational.

These examples suggest that the Gorenstein requirement might be necessary for Theorem 1.1 or 1.2

For a uniruled threefold $X$ with $-K_{X}$ big and an endomorphism, we have the following equivariant minimal model program (MMP); see Corollaries 4.1, 4.2 and Remarks 4.3, 4.4 for applications. As a referee remarked, the assertion (4) is well known and also follows from [21, Lemma 2.8].

Theorem 1.3. Let $X$ be a $\mathbb{Q}$-factorial projective threefold with only terminal singularities, a big $-K_{X}$ and a surjective endomorphism $f: X \rightarrow X$ of degree $q^{3}>1$. Let $X=X_{0} \cdots \rightarrow X_{1} \cdots \cdots \rightarrow X_{t}$ be a composition of $K_{X_{i}}$-negative flips and divisorial contractions such that there is no $K_{X_{t}}$-negative extremal contraction of birational type. Replacing $f$ by some power, we have (cf. also 4.4):

(1) For every $1 \leq j \leq t$, the dominant rational map $f_{j}: X_{j} \cdots \rightarrow X_{j}$ induced from $f$ is holomorphic. Set $f_{0}:=f$.

(2) There is a $K_{X_{t}}$-negative extremal contraction $X_{t} \rightarrow Y$ with $\operatorname{dim} Y \leq 2$. The morphism $f_{t}$ descends to a surjective endomorphism $h: Y \rightarrow Y$.

(3) Either $\operatorname{dim} Y \in\{1,2\}$, or $X_{t}$ is a $\mathbb{Q}$-Fano threefold of Picard number one (a consequence of $M M P)$. In the latter case, all $f_{i}(0 \leq i \leq t)$ are polarized (cf. 2.1(1)), and further

$$
f_{i}^{*} \mid N^{1}\left(X_{i}\right)=q \text { id } .
$$

(4) Suppose further that $(X, \Theta)$ is klt weak $\mathbb{Q}$-Fano for some $\Theta \geq 0$. Then for all $0 \leq i \leq t$, there are some $\Theta_{i} \geq 0$ such that the pairs $\left(X_{i}, \Theta_{i}\right)$ are klt $\mathbb{Q}$-Fano.

Remark 1.4. Theorem 1.2 was proved in 25 when the surjective morphism $f$ : $X \rightarrow X$ is assumed further to be polarized (cf. 2.1(1) below for its definition). The author does not know whether Theorem 1.2 follows from the result in [25, but being polarized is a strong condition (nevertheless, see also Theorem 1.3(3)). For instance, taking any endomorphism $g: Y \rightarrow Y$, then $f=g \times h: Y \times \mathbb{P}^{1} \rightarrow Y \times \mathbb{P}^{1}$ is not polarized if $(\operatorname{deg} h)^{\operatorname{dim} Y} \neq \operatorname{deg} g$ (cf. [20, Lemma 2.1]).

For an arbitrary uniruled variety $X$ with an endomorphism $f$, we would like to have an equivariant MMP: $X \cdots \rightarrow X_{m}$ to reduce to the Fano fibration case. To do so, we need to prove that the $K$-negative extremal rays appearing in the composition $X \cdots \rightarrow X_{m}$ of birational contractions are stabilized by $f$ and its descents. This is not easy to prove because the Mori cone $\overline{\mathrm{NE}}(X)$ may have infinitely many extremal rays and $X \cdots \rightarrow X_{m}$ may involve flips and hence may not be holomorphic. Fortunately, for Theorem 1.3, these difficulties are overcome by Theorem 2.16 .

We refer to [4, [5], [12, [18, 19, 20], 24], and [25] for the recent development in the study of the dynamics of endomorphisms of complex varieties. 


\section{Preliminary Results}

2.1. Conventions are as in Hartshorne's book, [9] and [10]. Some more:

(1) Every endomorphism $f$ of a projective variety in this paper is assumed to be surjective; so it is finite by the projection formula.

An endomorphism $f: X \rightarrow X$ is polarized if there is an ample Cartier integral divisor $H$ such that $f^{*} H \sim q H$ for some $q>0$. So $\operatorname{deg}(f)=q^{\operatorname{dim} X}$.

(2) Let $f: X \rightarrow X$ be an endomorphism and $\sigma_{V}: V \rightarrow X$ and $\sigma_{Y}: X \rightarrow Y$ morphisms. We say that $f$ lifts to an endomorphism $f_{V}: V \rightarrow V$ if $f \circ \sigma_{V}=\sigma_{V} \circ f_{V}$; $f$ descends to an endomorphism $f_{Y}: Y \rightarrow Y$ if $\sigma_{Y} \circ f=f_{Y} \circ \sigma_{Y}$.

(3) Every boundary divisor in a pair is assumed to be an effective $\mathbb{Q}$-divisor.

(4) A pair $(X, \Theta)$ is called a $k l t$ weak $\mathbb{Q}$-Fano variety (resp. klt $\mathbb{Q}$-Fano variety) if $X$ is a $\mathbb{Q}$-factorial normal projective variety, the pair $(X, \Theta)$ has at worst klt singularities as in [10, Definition 2.34] and $-\left(K_{X}+\Theta\right)$ is nef and big (resp. ample). When $\Theta=0$, the pair $(X, \Theta)$ is simply denoted as $X$. Thus $X$ is a klt weak $\mathbb{Q}$-Fano variety, or a $k l t \mathbb{Q}$-Fano variety if so is $(X, 0)$. A klt weak $\mathbb{Q}$-Fano variety (resp. a klt $\mathbb{Q}$-Fano variety) with $(X, \Theta)$ terminal (strengthened condition) is called a weak $\mathbb{Q}$-Fano variety (resp. a $\mathbb{Q}$-Fano variety).

(5) Suppose that $X$ is a normal projective variety and $(X, \Theta)$ is terminal or klt. An extremal ray in the Mori cone $\overline{\mathrm{NE}}(X)$, the nef cone $\operatorname{Nef}(X)$ or some other convex cones is in the sense of a locally polyhedral cone. A $\left(K_{X}+\Theta\right)$-negative extremal ray in $\overline{\mathrm{NE}}(X)$ is an extremal ray whose intersection with $K_{X}+\Theta$ is negative. By a $\left(K_{X}+\Theta\right)$-negative extremal contraction $X \rightarrow Y$, we mean the contraction of a $\left(K_{X}+\Theta\right)$-negative extremal ray in $\overline{\mathrm{NE}}(X)$. In particular, the Picard number $\rho(X)=\rho(Y)+1$. When $\Theta=0$, such a contraction is simply called an extremal contraction, or divisorial contraction, or flip, accordingly.

(6) By a conic bundle $X \rightarrow Y$, we mean an extremal contraction of relative dimension 1 , where $X$ is normal projective with only terminal singularities (so a general fibre is $\left.\mathbb{P}^{1}\right)$.

(7) For a surjective morphism $f: X \rightarrow Y$, the discriminant of $f$ is defined as $D(X / Y):=\{y \in Y ; f$ is not smooth over $y\}$. Let $D_{s}(X / Y)$ be the $s$-dimensional part of $D(X / Y)$, which is also regarded as a reduced scheme.

(8) For an endomorphism $\varphi: V \rightarrow V$ of a finite-dimensional real vector space $V, \rho(\varphi):=\max \{|\lambda| ; \lambda \in \mathbb{C}$ is an eigenvalue of $\varphi\}$ is the spectral radius.

$(9)$ For a $\mathbb{Q}$-factorial normal projective variety $X$, denote $S(X):=\{$ prime divisor $M ; M_{\mid M}$ is not pseudo-effective\}. When $\operatorname{dim} X=2$, our $S(X)$ is the set of negative curves on $X$.

(10) For a normal projective surface $X$, denote by Weil $(X)$ the finite-dimensional $\mathbb{R}$-vector space of Weil divisors modulo Mumford numerical equivalence: two Weil divisors are Mumford numerically equivalent if their Mumford pullbacks to a resolution are numerically equivalent.

Lemma 2.2(1) is from [16, Theorem 1.2.7]. For Lemma 2.2(2), see [14, Theorem (3.5)] (for the smooth case) and [16, Corollary 2.4.2] (originally due to Cutkosky).

Lemma 2.2. Let $X \rightarrow Y$ be a conic bundle with $(\operatorname{dim} X, \operatorname{dim} Y)=(3,2)$. Then we have:

(1) $Y$ has at worst Du Val singularities of type $A_{n}$.

(2) If $X$ is Gorenstein, then $Y$ is smooth. 
The following are known results about the rationality of threefolds; see [8, $\delta \S 2.2$ - 2.3], [13, §4.7] and the references therein.

Theorem 2.3. Let $X$ be a $\mathbb{Q}$-factorial projective threefold with at worst terminal singularities. Then $X$ is rational if either one of the following three conditions is satisfied.

(1) There is an extremal contraction $X \rightarrow B \cong \mathbb{P}^{1}$ such that a general fibre $F$ is a del Pezzo surface with $K_{F}^{2} \geq 5$.

(2) $X$ is smooth. There is an extremal contraction $X \rightarrow Y$ onto a rational surface $Y$, which is a non-standard conic bundle (cf. Remark 2.4).

(3) $X$ is smooth. There is an extremal contraction $X \rightarrow Y$ onto a rational surface $Y$, which is a standard conic bundle. Either $Y=\mathbb{P}^{2}$ and the discriminant $D:=D(X / Y)$ has $\operatorname{deg}(D) \leq 4$, or there is a $\mathbb{P}^{1}$-fibration on $Y$ such that $F . D \leq 3$ for a general fibre $F$.

Remark 2.4. $\quad$ (i) In (2) and (3) of Theorem 2.3, $Y$ is smooth (cf. Lemma 2.2). According to [13, 4.7], a conic bundle $\pi: X \rightarrow Y$ is standard if $\operatorname{Pic}(X)=$ $\pi^{*} \operatorname{Pic}(Y) \oplus \mathbb{Z} K_{X}$.

(ii) In Theorem 2.3(2), by [13, $\S 4.7]$, ' $X \rightarrow Y$ is non-standard' if and only if ' $X \rightarrow Y$ is a $\mathbb{P}^{1}$-bundle in the Zariski-topology'; such a $\mathbb{P}^{1}$-bundle is locally trivial and hence $X$ is rational, due to the triviality of the Brauer group $\operatorname{Br}(Y)$ for smooth rational surfaces.

(iii) Theorem 2.3(3) was proved by Iskovskikh [7, Theorem 1]; see his survey [8, $\S 2.3$, Theorem 8] for its variation and references.

For the first part of Lemma 2.5 below, see [13, Lemma 4.1, Remark 4.2]; the last part follows from the first part and the Riemann-Roch theorem as proved in [3. Lemma 2.3].

Lemma 2.5. Let $X \rightarrow Y$ be a conic bundle with $X$ smooth and $(\operatorname{dim} X, \operatorname{dim} Y)$ $=(3,2)$. Then the discriminant $D=D(X / Y)$ is a divisor of normal crossings, and every smooth rational component of $D$ (if it exists) meets at least two points of the other components. In particular, we have $\left|K_{Y}+D_{i}\right| \neq \emptyset$, when $Y$ is rational and $D_{i}$ is a connected component of $D$.

Lemma 2.6. Let $\pi: X \rightarrow Y$ be a conic bundle with $(\operatorname{dim} X, \operatorname{dim} Y)=(3,2)$ and $Y$ a rational surface. Then either the generic fibre is rational over $\mathbb{C}(Y)$ and hence $X$ is rational, or there is a conic bundle $\pi^{\prime}: X^{\prime} \rightarrow Y^{\prime}$ such that:

(1) $X^{\prime}$ (and hence $Y^{\prime}$ ) are smooth, and there are birational morphisms $\sigma_{x}$ : $X^{\prime} \rightarrow X$ and $\sigma_{y}: Y^{\prime} \rightarrow Y$ satisfying $\pi \circ \sigma_{x}=\sigma_{y} \circ \pi^{\prime}$.

(2) No connected component $C_{i}^{\prime}$ of $D\left(X^{\prime} / Y^{\prime}\right)$ is contracted to a point by $\sigma_{y}$.

(3) (The 1-dimensional part) $D_{1}(X / Y)=\sigma_{y *} D\left(X^{\prime} / Y^{\prime}\right) \neq \emptyset$.

(4) $D_{1}(X / Y)$ and $D\left(X^{\prime} / Y^{\prime}\right)$ have equal numbers of connected components.

Proof. (1) is actually proved in [13, Theorem 4.8]. If (2) is not true, $C_{i}^{\prime}$ is contracted to a point on $Y$ which is at worst of Du Val singularity of type $A_{n}$ (cf. Lemma 2.2) and hence $C_{i}^{\prime}$ is a rational tree, which contradicts Lemma 2.5. Part (3) is true because every reducible fibre over some $d \in D(X / Y)$ should be underneath only reducible fibres over some $d^{\prime} \in D\left(X^{\prime} / Y^{\prime}\right)$ and note that $\sigma_{y}: Y^{\prime} \rightarrow Y$ is the blowup along $D(X / Y)$; see the construction in [13, Theorem 4.8]; note also that $\left(\pi^{\prime}\right)^{*} E$ is irreducible for every prime divisor $E \subset Y^{\prime}$ (and especially for those $\left.E \subset D\left(X^{\prime} / Y^{\prime}\right)\right)$. Part (4) is from Parts (2) and (3). 
Proposition 2.7. Let $X \rightarrow Y$ be a conic bundle with $Y$ a rational surface. Suppose that either $D_{1}(X / Y)=\emptyset$, or there is a smooth rational curve $F$ on $\tilde{Y}$, with $\sigma$ : $\tilde{Y} \rightarrow Y$ a minimal resolution, such that $B s|F|=\emptyset$ and $F \cdot \sigma^{*} D_{1}(X / Y) \leq 3$. Then $X$ is rational.

Proof. We may assume that $D_{1}(X / Y) \neq \emptyset$ (cf. Lemma 2.6) and $F$ is a general member in $|F|$. Let $Y^{\prime \prime} \rightarrow \widetilde{Y}$ be the blowup of $m=F^{2}$ points on $F$ away from $F \cap$ $\sigma^{-1} D(X / Y)$. Take a blowup $X^{\prime \prime} \rightarrow X$ so that the composite $X^{\prime \prime} \rightarrow X \rightarrow Y \cdots \rightarrow Y^{\prime \prime}$ extends to a morphism with general fibre $\mathbb{P}^{1}$. By the proof of [13, Theorem 4.8], we have a lifting $\pi^{\prime}: X^{\prime} \rightarrow Y^{\prime}$ of $\pi$ as in Lemma 2.6 so that $Y^{\prime} \rightarrow Y$ factors as $Y^{\prime} \rightarrow Y^{\prime \prime} \rightarrow \widetilde{Y} \rightarrow Y$. Denote by $D^{\prime}=D\left(X^{\prime} / Y^{\prime}\right)$ and by $D^{\prime \prime}, \widetilde{D}, D$ its pushforwards (as cycles) on $Y^{\prime \prime}, \tilde{Y}$ and $Y$. Note that $D=D_{1}(X / Y)$ by Lemma 2.6.

Claim 2.8. $\sigma^{*} D \geq \widetilde{D}$.

We prove Claim 2.8. Write $\sigma^{*} D-\widetilde{D}=\sum_{i} e_{i} E_{i}$ with $E_{i}$ irreducible and $\sigma$ exceptional. Lemma 2.2 implies that each $E_{i}$ is a $(-2)$-curve. By Zariski's lemma, we have only to show the assertion that $0 \geq E_{j} \cdot\left(\sigma^{*} D-\widetilde{D}\right)=-E_{j} . \widetilde{D}$ for every $E_{j}$. We need to consider the case where $E_{j}$ is a component of $\widetilde{D}$. Applying Lemma 2.5 to $D^{\prime}$ and using Lemma 2.6, we have $E_{j} . \widetilde{D} \geq 0$. This proves Claim 2.8,

We continue the proof of Proposition 2.7 The proper transform $F^{\prime \prime} \subset Y^{\prime \prime}$ of $F$ is a fibre of a $\mathbb{P}^{1}$-fibration. Let $F^{\prime} \subset Y^{\prime}$ be the total transform of $F^{\prime \prime}$. Then $F^{\prime} . D^{\prime}=F^{\prime \prime} . D^{\prime \prime}=F \cdot \widetilde{D} \leq F \cdot \sigma^{*} D \leq 3$. Now Proposition 2.7 follows from Theorem 2.3 .

Lemma 2.9. Let $X \rightarrow Y$ be a conic bundle with $\operatorname{dim} Y=2$, and $f: X \rightarrow X$ a surjective endomorphism descending to an endomorphism $h: Y \rightarrow Y$. Then $h^{-1}(D)=D$ for the (1-dimensional part) $D:=D_{1}(X / Y)$, so after replacing $f$ by some power, we have $h^{-1}\left(D_{i}\right)=D_{i}$ for every irreducible component $D_{i}$ of $D$.

Proof. We have $h^{-1}(D) \subseteq D$ since the reducibility of a fibre $X_{d}$ over $d \in D$ implies that of $X_{d^{\prime}}$ for every $d^{\prime} \in h^{-1}(d)$. So $D \supseteq h^{-1}(D) \supseteq h^{-2}(D) \supseteq \cdots$. Considering the number of irreducible components, we have $h^{-s}(D)=h^{-s-1}(D)$ for some $s>1$. Since $h$ is surjective and applying $h^{s}$ and $h^{s+1}$ we get $h^{ \pm}(D)=D$. This proves the lemma.

The result below can be proved as in [17, Lemma 8 and Proposition 10]. The assumption on $X_{i}$ can be weakened to being normal Moishezon by using the Mumford intersection on surfaces.

Lemma 2.10. Let $\varphi: X_{1} \rightarrow X_{2}$ be a finite morphism between two $\mathbb{Q}$-factorial normal projective surfaces with the same Picard number $\rho\left(X_{1}\right)=\rho\left(X_{2}\right)$. Then we have:

(1) $\varphi$ induces a bijection $\varphi: S\left(X_{1}\right) \rightarrow S\left(X_{2}\right)(C \mapsto \varphi(C))$ with a well-defined inverse mapping $C^{\prime}$ to $\varphi^{-1}\left(C^{\prime}\right)$. Further, $\varphi^{*} C_{2}=\alpha C_{1}$, whenever $C_{2} \in$ $S\left(X_{2}\right), C_{1}=\varphi^{-1}\left(C_{2}\right)$ and $\alpha=\sqrt{\operatorname{deg}(\varphi) C_{2}^{2} / C_{1}^{2}}$.

(2) Suppose that $\operatorname{deg}(\varphi)>1$. Then $\left|S\left(X_{i}\right)\right|<\infty$; we may also assume that $\varphi \mid S\left(X_{1}\right)=\mathrm{id}$, when $\varphi: X_{1} \rightarrow X_{2}=X_{1}$ is an endomorphism and $\varphi$ is replaced by some power. 
As a referee remarked, Lemma 2.11 and Lemma 2.12(2) are well known and the latter also follows from [21, Lemma 2.8]. We give proofs for them for the convenience of readers.

Lemma 2.11. Let $(X, \Theta)$ be a klt weak $\mathbb{Q}$-Fano variety. Then we have:

(1) $\left(X, \Theta_{0}\right)$ is $k l t \mathbb{Q}$-Fano for some $\Theta_{0} \geq \Theta$.

(2) The cone $\overline{\mathrm{NE}}(X)$ has only finitely many extremal rays $\left(K_{X}\right.$ negative or non-negative).

(3) $X$ is rationally connected.

Proof. (3) is proved in [26, Theorem 1]. For (1), by [10, Proposition 2.61], $-\left(K_{X}+\right.$ $\Theta) \sim_{\mathbb{Q}} A_{k}+\frac{1}{k} E$ for some ample $\mathbb{Q}$-divisor $A_{k}$ and effective $\mathbb{Q}$-divisor $E$. Choose $k \gg 0$ so that $\left(X, \Theta+\frac{1}{k} E\right)$ has at worst klt singularities; see [10, Corollary 2.35]. Now $-\left(K_{X}+\Theta+\frac{1}{k} E\right) \sim_{\mathbb{Q}} A_{k}$ is ample. (1) is proved. (2) is true by the cone theorem as in [10, Theorem 3.7].

Lemma 2.12. (1) Let $\varphi: V \rightarrow W$ be a generically finite surjective morphism between projective varieties. Suppose that the cone $\overline{\mathrm{NE}}(V)$ has only finitely many extremal rays $\mathbb{R}_{\geq 0}\left[C_{i}\right]$ ( $K_{V}$ negative or non-negative). Then $\overline{\mathrm{NE}}(W)$ has only finitely many extremal rays, each of which is generated by some $\varphi\left(C_{i}\right)$.

(2) Suppose that $(V, \Theta)$ is a klt weak $\mathbb{Q}$-Fano variety. Let $\sigma: V \cdots \rightarrow W$ be a $K_{V}$-flip or a $K_{V}$-divisorial contraction. Then $\left(W, \Theta_{W}\right)$ is $k l t \mathbb{Q}$-Fano for some $\Theta_{W} \geq 0$. Hence both cones $\overline{\mathrm{NE}}(V)$ and $\overline{\mathrm{NE}}(W)$ have only finitely many extremal rays. (We remark that if $V$ is terminal, then so is $W$; cf. [10, Corollary 3.42]).

Proof. (1) is true, since $\varphi$ induces a surjective additive map $\varphi_{*}: \overline{\mathrm{NE}}(V) \rightarrow \overline{\mathrm{NE}}(W)$.

For (2), consider the case where $\sigma$ is a flip. Let $\pi: V \rightarrow Y$ and $\pi^{+}: W=$ $V^{+} \rightarrow Y$ be the flipping contractions. Let $H$ be an ample $\mathbb{Q}$-divisor on $Y$. By Lemma 2.11, we may assume that $-\left(K_{V}+\Theta\right)$ is ample after replacing $\Theta$. Thus $-m\left(K_{V}+\Theta\right)-\pi^{*} H$ is very ample for $m \gg 0$ and hence linearly equivalent to an irreducible divisor $G_{m}$. Then $\left(V, \Theta+\frac{1}{m} G_{m}\right)$ is klt by [10, Corollary 2.31]. Let $\Theta_{W}^{\prime} \subset W$ be the proper transform of $\Theta+\frac{1}{m} G_{m}$. Then $W$ is $\mathbb{Q}$-factorial and $\left(W, \Theta_{W}^{\prime}\right)$ is klt by [10, Proposition 3.37, Corollary 3.42]. Also $-\left(K_{W}+\Theta_{W}^{\prime}\right)$ is $\mathbb{Q}$-linearly equivalent to the nef and big divisor $\left(\pi^{+}\right)^{*} H / m$. Now (2) follows from Lemma 2.11,

Consider the case where $\sigma$ is divisorial. By Lemma 2.11 we may assume that $(V, \Theta)$ is klt $\mathbb{Q}$-Fano. Take a small ample $\mathbb{Q}$-divisor $H_{W} \subset W$ such that $-\left(K_{V}+\right.$ $\Theta)-\sigma^{*} H_{W}$ is still ample and hence it is $\mathbb{Q}$-linearly equivalent to some $\Delta>0$ with $(V, \Theta+\Delta)$ klt by the argument in the previous case. Then $-\left(K_{V}+\Theta+\Delta\right) \sim_{\mathbb{Q}} \sigma^{*} H_{W}$. Set $\Theta_{W}:=\sigma_{*}(\Theta+\Delta)$. Now $-\left(K_{W}+\Theta_{W}\right) \sim_{\mathbb{Q}} \sigma_{*} \sigma^{*} H_{W}=H_{W}$, which is ample, and $K_{V}+\Theta+\Delta \sim_{\mathbb{Q}} \sigma^{*}\left(K_{W}+\Theta_{W}\right)$. Thus $\left(W, \Theta_{W}\right)$ is klt (and $\mathbb{Q}$-Fano) because so is $(V, \Theta+\Delta)$. This proves the lemma.

Below is Birkhoff's generalization of the Perron-Frobenius theorem (cf. 2]).

Theorem 2.13. Let $C$ be a strictly convex closed cone of a finite-dimensional real vector space $V$ such that $C$ generates $V$ as a vector space. Let $\varphi: V \rightarrow V$ be an endomorphism such that $\varphi(C) \subseteq C$. Then the spectral radius $\rho(\varphi)$ is an eigenvalue of $\varphi$ and corresponds to an eigenvector in $C \backslash\{0\}$. 
Lemma 2.14. Let $V$ be a normal projective surface and $f: V \rightarrow V$ a surjective endomorphism. Then $\operatorname{deg}(f) \leq \rho\left(f^{*}\right)^{2}$, where $\rho\left(f^{*}\right)$ is the spectral radius of $f^{*} \mid \operatorname{Weil}(V)$.

Proof. By Theorem 2.13, there is a non-zero $\mathbb{R}$-Cartier nef divisor $L \in$ Weil $(V)$ such that $f^{*} L \equiv \rho L$, where $\rho:=\rho\left(f^{*}\right)$. Take a Jordan canonical basis $\left\{L_{i}\right\}_{i \geq 1}$ for $f^{*} \mid \operatorname{Weil}(V)$. Write $f^{*} L_{i}=\lambda_{i} L_{i}+L_{i-1}\left(\right.$ set $\left.L_{0}:=0\right)$. Note that $\left|\lambda_{i}\right| \leq \rho$. We may assume that $L . L_{i}=0$ for all $i<s$ and $L . L_{s} \neq 0$. Now $\operatorname{deg}(f) L . L_{s}=f^{*} L . f^{*} L_{s}=$ $\rho L .\left(\lambda_{s} L_{s}+L_{s-1}\right)=\rho \lambda_{s} L . L_{s}$. So $\operatorname{deg}(f)=\rho \lambda_{s} \leq \rho^{2}$.

Lemma 2.15. Let $X=X_{0} \cdots \rightarrow X_{1} \cdots \cdots \rightarrow X_{r}$ be a composition of flips and divisorial contractions corresponding to $\left(K_{X_{i}}+\Theta_{i}\right)$-negative extremal rays on some $\mathbb{Q}$-factorial klt pairs $\left(X_{i}, \Theta_{i}\right)$. Suppose that $f=f_{0}: X \rightarrow X$ is a surjective endomorphism and descends to surjective endomorphisms $f_{i}: X_{i} \rightarrow X_{i}(1 \leq i \leq r)$. Suppose further that for some $0 \leq k \leq r$ we have $\left(f_{k}^{s_{k}}\right)^{*} \mid N^{1}\left(X_{k}\right)=q^{s_{k}}$ id for some $q>1$ and integer $s_{k}>0$. Then all $f_{j}(0 \leq j \leq r)$ are polarized, and $\left(f_{j}^{s}\right)^{*} \mid N^{1}\left(X_{j}\right)=q^{s}$ id for some integer $s>0$.

Proof. Let $H^{\prime} \subset X_{k}$ be an ample $\mathbb{Q}$-divisor and set $H:=\sum_{i=0}^{s_{k}-1}\left(f_{k}^{i}\right)^{*} H^{\prime} / q^{i}$. Then $f_{k}^{*} H \equiv q H$. Thus $f_{k}$ is polarized by [20, Lemma 2.2].

By the remark above, we only need to show the second assertion, and for that we will prove by the ascending induction on $|k-i|$. We may assume that $s_{k}=1$, after replacing $f$ by some power. It suffices to show that $f_{i}^{*} \mid N^{1}\left(X_{i}\right)=q$ id for $i=k-1$ and $k+1$. So we may assume that $(k, r)=(0,1)$ or $(1,1)$.

Case 1. $\sigma: X \rightarrow X_{1}$ is a divisorial contraction with $E$ the exceptional divisor. $E$ is irreducible by [9, Proposition 5-1-6], and $f^{*} E=e E$ for some $e>0$ because both $f$ and its descent $f_{1}$ are necessarily finite morphisms. If $k=0$ so that $f_{i}^{*} \mid N^{1}\left(X_{i}\right)=$ $q$ id for $i=0$, then the same holds for $i=1$ because $\sigma^{*} N^{1}\left(X_{1}\right)$ is a subspace of $N^{1}(X)$ and $f_{1}$ is the descent of $f$. Suppose that $k=1$ so that $f_{1}^{*} \mid N^{1}\left(X_{1}\right)=q$ id. Then $f^{*} \mid N^{1}(X)=\operatorname{diag}[e, q, \ldots, q]$ with respect to a basis consisting of $E$ and a basis of $\sigma^{*} N^{1}\left(X_{1}\right)$. For an ample divisor $H^{\prime} \subset X_{1}$, the divisor $H:=\sigma^{*} H^{\prime}$ is nef and big and $f^{*} H \equiv q H$, so $e=q$ by [20, Lemma 2.1]. Thus $f^{*} \mid N^{1}(X)=q$ id. We are done.

Case 2. $\sigma: X \cdots \rightarrow X_{1}$ is a flip. Then $\sigma$ induces an isomorphic linear map between $N^{1}(X)$ and $N^{1}\left(X_{1}\right)$ compatible with the two actions $f_{i}^{*} \mid N^{1}\left(X_{i}\right)$ with $i=0,1$; see the proof of [10, Proposition 3.37]. So the scalarity of one action implies that of the other. This proves the lemma.

The theorem below is the key in proving Theorem 1.3

Theorem 2.16. Let $X$ be a $\mathbb{Q}$-factorial projective threefold with only terminal singularities, big $-K_{X}$ and a surjective endomorphism $f: X \rightarrow X$ of degree $d>1$. Let $\mathbb{R}_{\geq 0}[\ell]$ be a $K_{X}$-negative extremal ray. Then, replacing $f$ by some power, we have:

(1) $f(\ell)$ is parallel to $\ell$, i.e., $f(\ell) \in \mathbb{R}_{>0}[\ell]$.

(2) Suppose that $\mathbb{R}_{\geq 0}[\ell]$ gives rise to a divisorial contraction $\sigma: X \rightarrow X_{1}$, or a flip $\sigma: X \cdots \rightarrow X^{+}=: X_{1}$, or a Fano fibration $X \rightarrow X_{1}$ with $\operatorname{dim} X_{1} \leq 2$. Then $f$ descends to a surjective endomorphism of $X_{1}$. Further, $-K_{X_{1}}$ is also big when $\sigma$ is divisorial or a flip. 
Proof. We only need to show the first assertion of Theorem 2.16 because the second follows from the first; see [25, Lemmas 2.12 and 3.6]. Indeed, the bigness of $-K_{X}$ implies that of $-K_{X_{1}}=\sigma_{*}\left(-K_{X}\right)$ when $\sigma$ is divisorial; $-K_{X_{1}}$ is also big when $\sigma$ is a flip, because $\sigma$ is then isomorphic in codimension one.

We now prove Theorem 2.16(1). We may assume that $\ell$ is an irreducible curve. Write $-K_{X}=A+D$ with $A$ an ample $\mathbb{Q}$-divisor and $D$ an effective $\mathbb{Q}$-divisor. By [10, Theorem 3.7], for every $1>\varepsilon>0$, the first assertion below is true.

Claim 2.17. (1) The subcone $R_{\varepsilon}:=\overline{\mathrm{NE}}(X)_{K_{X}+\varepsilon A<0}$ of $\overline{\mathrm{NE}}(X)$ contains only finitely many extremal rays.

(2) Suppose that $\mathbb{R}_{\geq 0}[\ell]$ gives rise to a divisorial contraction $\sigma: X \rightarrow X_{1}$ with $E$ the exceptional (necessarily prime) divisor and $\ell$ taken to be a fibre of $\sigma$. Then the subspace $N^{1}(X) . E \subset N_{1}(X)$ has rank $\leq 2$, and the ray $\mathbb{R}_{\geq 0}[\ell]$ is extremal in (the closure of) the cone $\operatorname{Nef}(X) . E \subset N_{1}(X)$.

For Claim 2.17(2), if $\sigma(E)$ is a point, then $N^{1}(X) \cdot E=\mathbb{R}[\ell]$, and we are done! Suppose that $\sigma(E)$ is a curve. For any divisor $M$ on $X$ we have $(M-a A) \cdot \ell=0$ for some $a \in \mathbb{R}$ and hence $M-a A=\sigma^{*} L$ for some divisor $L$ on $Y_{1}$; see [9, Lemma $3-2-5]$. Then $M . E=a A . E+b \ell$ with $b=L . \sigma(E)$. Thus $N^{1}(X) . E$ is spanned by $A$.E and $\ell$. Claim $2.17(2)$ is true because the ray there, is extremal in the bigger cone $\overline{\mathrm{NE}}(X)$. This proves Claim 2.17 .

We return to the proof of Theorem 2.16.

Note that every $\mathbb{R}_{\geq 0}\left[f^{s}(\ell)\right](s \in \mathbb{Z})$ is an extremal ray in $\overline{\mathrm{NE}}(X)\left(K_{X}\right.$ negative or non-negative); see [25, Lemma 2.11]. If $f^{s}(\ell)$ is contained in $R_{\varepsilon}$ for infinitely many $s=s_{1}, s_{2}, \ldots$, then by Claim 2.17, we may assume that $f^{s_{1}}(\ell)$ is parallel to $f^{s_{2}}(\ell)$ for some $s_{2}>s_{1}$. Thus $f^{s_{2}-s_{1}}(\ell)$ is parallel to $\ell$ because $f_{*}$ is an automorphism of the vector space $N_{1}(X)$. We are done.

Therefore, we may assume that $f^{s}(\ell)$ is not contained in $R_{\varepsilon}$ for all $s \in \mathbb{Z} \backslash\{0\}$ after $f$ is replaced by some power. Note that $\bigcup_{s>0} f^{s}(\ell) \subset D$ because:

$$
\begin{aligned}
-\left(K_{X}+\varepsilon A\right) & =(1-\varepsilon) A+D, \\
0 \geq-f^{s}(\ell) \cdot\left(K_{X}+\varepsilon A\right) & =(1-\varepsilon) f^{s}(\ell) \cdot A+f^{s}(\ell) \cdot D>f^{s}(\ell) \cdot D .
\end{aligned}
$$

Case 1. $R_{\geq 0}[\ell]$ gives rise to a Fano fibration $\sigma: X \rightarrow X_{1}$ with $\operatorname{dim} X_{1} \leq 2$ and $\ell$ chosen to be in a general fibre of $\sigma$. Then $f(\ell)$ is not contained in $D$. This is a contradiction.

Case 2. $R_{\geq 0}[\ell]$ gives rise to a divisorial contraction $\sigma: X \rightarrow X_{1}$ with $E$ the exceptional (prime) divisor and $\ell$ taken as a fibre of $\sigma$. Since $f^{s}(\ell) . D<0(s \neq 0)$, we have $f(\ell) . D_{1}<0$ for an irreducible component $D_{1}$ of $D$. Every fibre $\ell^{\prime}$ of $\sigma$ is parallel to $\ell$, so $f\left(\ell^{\prime}\right) . D_{1}<0$. Thus $f\left(\ell^{\prime}\right) \subset D_{1}$ and hence $f(E)=D_{1}$. Now $f^{2}(\ell) . D<0$ implies that $f^{2}(\ell) \cdot D_{j}<0$ for some irreducible component $D_{j}$ of $D$. As argued above, $f^{2}(E)=D_{j}$. Since $D$ has only finitely many components, we have $f^{r_{2}}(E)=f^{r_{1}}(E)$ for some $r_{2}>r_{1}$. Thus for fibres $\ell_{t}$ of $\sigma$, we have $f^{r_{2}}\left(\ell_{t}\right)=f^{r_{1}}\left(m_{t}\right)$ for some irreducible curve $m_{t} \subset E$. So $f^{r}\left(\ell_{t}\right)$ (and hence $f^{r}(\ell)$ ) is parallel to $m_{t}$, where $r:=r_{2}-r_{1}>0$. For an extremal curve $\ell^{\prime \prime}$, denote

$$
\Sigma_{\ell^{\prime \prime}}:=\left\{C \subset X ;[C] \in \mathbb{R}_{\geq 0}\left[\ell^{\prime \prime}\right]\right\}, \quad U_{\ell^{\prime \prime}}:=\bigcup_{C \in \Sigma_{\ell^{\prime \prime}}} C,
$$


where these $C$ are irreducible curves. Then $E=U_{\ell}$. By [25, Lemma 2.11],

$$
f^{r}(E)=f^{r}\left(U_{\ell}\right)=U_{f^{r}(\ell)} \supset \bigcup_{t} m_{t} \subset E .
$$

The map $\left\{\ell_{t}\right\} \rightarrow\left\{m_{t}\right\}$ is a finite-to-finite map. So $\left\{m_{t}\right\}$ is an infinite set and hence

$$
f^{r}(E)=\overline{\bigcup_{t} m_{t}}=E .
$$

By Claim 2.17, (the closure of) $\operatorname{Nef}(X) . E$ is generated by one or two extremal rays (one being $\mathbb{R}_{\geq 0}[\ell]$ ) each of which is preserved by $f_{*}^{2 r}$. Thus $f^{2 r}(\ell)$ is parallel to $\ell$. We are done.

Case 3. $\mathbb{R}_{\geq 0}[\ell]$ gives rise to a flip $X \cdots \rightarrow X^{+}$with flipping contractions $\pi: X \rightarrow Y_{1}$ and $\pi^{+}: \bar{X}^{+} \rightarrow Y_{1}$. We may assume that $f^{i}(\ell)$ and $f^{j}(\ell)$ are not parallel for all $i \neq j$. In particular, $f^{i}(\ell)$ 's are pairwise distinct. Replacing $f$ by some power, we may assume that

$$
\bigcup_{s>0} f^{s}(\ell) \subset \bigcup_{i=1}^{r} D_{i} \subseteq \operatorname{Supp} D
$$

and $I_{j}:=\left\{s>0 \mid f^{s}(\ell) \subset D_{j}\right\}$ is an infinite set for all $1 \leq j \leq r$. Thus

$$
D_{j}=\overline{\bigcup_{s \in I_{j}} f^{s}(\ell)}
$$

Each $\Sigma_{f^{s}(\ell)}=f^{s}\left(\Sigma_{\ell}\right)$ is a union of finitely many (and, when $s \gg 0$, the same number of) curves. Since $f^{-s}\left(\Sigma_{f^{s}(\ell)}\right)=\Sigma_{\ell}$ (cf. 25, Lemma 2.11]), replacing $\ell$ by some $f^{s}(\ell)$, we may assume that for $\ell_{i}:=f^{i}(\ell)(i \geq 0)$ we have $f^{-1}\left(\ell_{i+1}\right)=\ell_{i}$ and hence $f^{-i} f^{i}\left(\ell_{0}\right)=\ell_{0}$. Now $f^{ \pm}$permutes the $D_{j}$ 's. Indeed,

$$
f\left(D_{1}\right)=f\left(\overline{\bigcup_{s \in I_{1}} f^{s}(\ell)}\right)=\overline{\bigcup_{s \in I_{1}} f^{s+1}(\ell)} \subset \bigcup_{i=1}^{r} D_{i} .
$$

Hence $f\left(D_{1}\right)=D_{j_{0}}$ for some $j_{0}$. Also, by the choice of $j_{0}$ and $\ell_{0}$,

$$
f^{-1}\left(D_{j_{0}}\right)=f^{-1} \overline{\bigcup_{s \in I_{1}} f^{s+1}(\ell)}=\overline{\bigcup_{s \in I_{1}} f^{-1} f^{s+1}(\ell)}=\overline{\bigcup_{s \in I_{1}} f^{s}(\ell)}=D_{1} .
$$

Replacing $f$ by some power, we may assume that $f^{ \pm}\left(D_{j}\right)=D_{j}$ for all $1 \leq j \leq r$, and also $f(\ell) \cdot D_{1}<0$ after relabelling, noting that only finitely many $f^{s}(\ell)$ are in $D \backslash \sum_{i=1}^{r} D_{i}$. In particular, $f(\ell) \subset D_{1}$ and hence $D_{1} \mid D_{1} \neq 0$. Write $f^{*} D_{1}=e_{1} D_{1}$ for some integer $e_{1}>0$. Then $f^{*}\left(D_{1} \mid D_{1}\right)=e_{1} D_{1} \mid D_{1}$.

Consider the case $\operatorname{deg}\left(f \mid D_{1}: D_{1} \rightarrow D_{1}\right)=1$. Then $e_{1}=d>1$. We claim that $f \mid D_{1}$ induces an automorphism $h$ of positive entropy on the normalization $\widetilde{D_{1}}$, the pullback of $-D_{1} \mid D_{1}$ is a nef eigenvector of $h^{*}$ and $e_{1}$ equals the spectral radius $\rho\left(h^{*}\right)$ of $h^{*} \mid \operatorname{NS}\left(\widetilde{D_{1}}\right)$. Indeed, applying Theorem 2.13 to the action of $h^{*}$ on the nef cone of $\widetilde{D_{1}}$, we have $h^{*} L=\rho\left(h^{*}\right) L$ for some non-zero $\mathbb{R}$-Cartier nef divisor $L$; thus the pullback of $-D_{1} \mid D_{1}$ is a positive multiple of $L$ by the uniqueness of the eigenvalue of $h^{*}$ of modulus $>1$ as in [12, Theorem 3.2] applied to an equivariant desingularization of $D_{1}$. The claim is proved.

On the other hand, $e_{1}$ (being the spectral radius) must be a Salem number (and an algebraic integer of degree $\geq 2$ over $\mathbb{Q}$ ) and $e_{1}$ and $e_{1}^{-1}$ are roots of their common minimal polynomial (over $\mathbb{Q}$ ) dividing the characteristic polynomial of $h^{*} \mid \operatorname{NS}\left(\widetilde{D_{1}}\right)$; 
see the proof of [12, Theorem 3.2] or [23, Proposition 2.11]. Indeed, $e_{1} \in \mathbb{N}$ and hence $\left(x-e_{1}\right)$ is the minimal polynomial of $e_{1}$ over $\mathbb{Q}$, which is of degree one, which is absurd!

Therefore, $f \mid D_{1}: D_{1} \rightarrow D_{1}$ has degree $d_{1}>1$. Then $d=d_{1} e_{1}$. Our $f \mid D_{1}$ induces a degree- $d_{1}$ endomorphism $h$ of the normalization $\widetilde{D_{1}}$ of $D_{1}$. After relabeling, we may assume that no $\ell_{i}=f^{i}(\ell)(i \geq 0)$ is included in the non-normal locus of $D_{1}$ or the image of the ramification divisor $R_{h}$. Denote by $\widetilde{\ell_{i}} \subset \widetilde{D_{1}}$ the pullback of $\ell_{i}$. Then we have $h^{*} \widetilde{\ell_{j+1}}=\widetilde{\ell_{j}}$ for all $j \geq 0$. Let $N$ be the denominator of the intersection matrix of the exceptional curves of a resolution of $D_{1}$. Then the Mumford intersection satisfies $C_{1} . C_{2} \in \frac{1}{N^{2}} \mathbb{Z}$ for all curves $C_{i}$ on $\widetilde{D_{1}}$. Now

$$
d_{1} \widetilde{\ell}_{i} \cdot \widetilde{\ell_{j}}=h^{*}{\widetilde{\ell_{i}}}_{i} \cdot h^{*}{\widetilde{\ell_{j}}}^{=\widetilde{\ell_{i-1}} \cdot \widetilde{\ell_{j-1}}}, \quad \frac{1}{N^{2}} \mathbb{Z} \ni \widetilde{\ell_{i}} \cdot \widetilde{\ell_{j}}=\frac{1}{d_{1}^{a}} \widetilde{\ell_{i-a}} \cdot \widetilde{\ell_{j-a}} .
$$

Applying the above to $i=j=a \gg 0$ or $i=j-1=a \gg 0$, we get

$$
\tilde{\ell}_{i}^{2}={\widetilde{\ell_{i+1}}}^{2}=\widetilde{\ell_{i}} \cdot \widetilde{\ell_{i+1}}=0 \text {. }
$$

Thus $\widetilde{\ell_{i+1}}$ is parallel to $\widetilde{\ell}_{i}$, by pulling back to a resolution of $D_{1}$ and applying the Hodge index theorem. So $\ell_{i+1}=f^{i+1}(\ell)$ is parallel to $\ell_{i}=f^{i}(\ell)$. Hence $f(\ell)$ is parallel to $\ell$. This proves Theorem 2.16.

Lemma 2.18. Let $X=X_{0}$ and $X_{1}$ be $\mathbb{Q}$-factorial projective threefolds with only terminal singularities and $f=f_{0}: X \rightarrow X$ and $f_{1}: X_{1} \rightarrow X_{1}$ surjective endomorphisms of the same degree $d>1$. Suppose that either $\sigma: X \rightarrow X_{1}$ is a $K_{X}$-divisorial contraction or $\sigma: X \cdots \rightarrow X_{1}$ is a $K_{X}$-flip, and that $f_{1}$ is the descent of $f$ in either case. Then the ramification divisor $R_{f}=0$, if and only if $R_{f_{1}}=0$, if and only if $f_{i}$ is étale over $X_{i} \backslash \operatorname{Sing} X_{i}$ for both $i$.

Proof. Note that the second part follows from the first and the purity of the branch loci. If $\sigma: X \cdots \rightarrow X_{1}$ is a flip, then $\sigma$ switches $R_{f}$ and $R_{f_{1}}$ because $\sigma$ is an isomorphism in codimension 1 and $f_{1}$ is the descent of $f$, so $R_{f}=0$ if and only if $R_{f_{1}}=0$.

Consider the case where $\sigma: X \rightarrow X_{1}$ is divisorial with $E$ the exceptional (prime) divisor. Then $\sigma_{*} R_{f}=R_{f_{1}}$, so $R_{f}=0$ implies $R_{f_{1}}=0$.

Suppose the contrary that $R_{f_{1}}=0$ and $R_{f} \neq 0$. Then $R_{f}=(e-1) E$, where $f^{*} E=e E$ with $e \geq 2$. Take a fibre $\ell$ of $\sigma$. Then $\ell . K_{X}<0$. It is well known that $\ell . E<0$. Since $f$ and $f_{1}$ are compatible, $f^{*} \ell \equiv c \ell$ for some $c>0$. Now $d \ell . E=f^{*} \ell . f^{*} E=c e \ell . E$ implies that $c=d / e$. Thus $f_{*} \ell=(d / c) \ell=e \ell$. Now multiplying the equality $K_{X}=f^{*} K_{X}+(e-1) E$ by $\ell$ and by the projection formula, we obtain

$$
\ell . K_{X}=\ell . f^{*} K_{X}+(e-1) \ell . E=e \ell . K_{X}+(e-1) \ell . E .
$$

Thus $0<-\ell . K_{X}=\ell . E<0$, which is absurd. So $R_{f}=0$ if and only if $R_{f_{1}}=0$.

\section{Fibration-PRESERVING ENDOMORPHiSMS}

In this section, we shall prove three lemmas used in the proof of the main theorems.

Remark 3.1. Every normal projective variety $Y$ of dimension $\leq 2$ and dominated by a rationally connected normal projective variety $X$ is rational. 
Lemma 3.2. Let $X$ be a rationally connected projective threefold with only terminal singularities, and $\pi: X \rightarrow Y$ a conic bundle. Suppose that $f: X \rightarrow X$ is an endomorphism of degree $d>1$ and it descends to an automorphism $h: Y \rightarrow Y$. Then $X$ is rational.

Proof. By Proposition 2.7, it suffices to show the claim below.

Claim 3.3. The (1-dimensional part) $D:=D_{1}(X / Y)$ is an empty set.

We prove the claim. Suppose to the contrary that $D \neq \emptyset$. Replacing $f$ by some power, we may assume, as in Lemma 2.9 that $h^{-1}\left(D_{i}\right)=D_{i}$ for every irreducible component $D_{i}$ of $D$. Thus $h^{*} D_{i}=D_{i}$ and $h^{*} D=D$ because $\operatorname{deg}(h)=1$.

Let $F$ be a very ample divisor on $Y$ away from $\Sigma:=D_{0}(X / Y) \cup \operatorname{Sing}(D) \cup$ $\pi(\operatorname{Sing} X) \cup \operatorname{Sing}(Y)$ and $h(\Sigma)$. Set $s:=F . D>0$. Since the Picard number $\rho(X / Y)=1, X_{F}:=\pi^{*} F$ is irreducible and is a smooth ruled surface (admitting a ruling $X_{F} \rightarrow F$ ) with $s$ singular fibres $S_{i}$ lying over the $s$ points $P_{i}$ in $D \cap F$. Further, each $S_{i}$ consists of two intersecting lines $S_{i}(1)+S_{i}(2)$ (see [14, or [13, $\S 4.1, \S 4.8])$.

Note that the Picard number $\rho\left(X_{F}\right)=2+s$. Denote $F^{\prime}=h^{*} F$. We have $f^{*} X_{F}=X_{F^{\prime}}:=\pi^{*} F^{\prime}$, which is also a smooth ruled surface with a ruling $X_{F^{\prime}} \rightarrow F^{\prime}$. Our $f$ restricts to a finite morphism $f \mid X_{F^{\prime}}: X_{F^{\prime}} \rightarrow X_{F}$ of degree $d$ while the latter descends to the isomorphism $h \mid F^{\prime}: F^{\prime} \rightarrow F$.

Now $D . F^{\prime}=h^{*} D \cdot h^{*} F=D . F=s$. So $X_{F^{\prime}}$ is a smooth ruled surface with the Picard number $\rho\left(X_{F^{\prime}}\right)=2+s=\rho\left(X_{F}\right)$. By Lemma 2.10, we have $\left(f \mid X_{F^{\prime}}\right)^{*}\left(S_{i}(j)\right)$ $=d_{i j} S_{i}(j)^{\prime}$ for some $d_{i j} \in \mathbb{N}$, where $S_{i}(1)^{\prime}+S_{i}(2)^{\prime}=\left(f \mid X_{F^{\prime}}\right)^{*} S_{i}$ are the $s$ singular fibres of the ruling $X_{F^{\prime}} \rightarrow F^{\prime}$ lying over the $s$ points $P_{i}^{\prime}=D \cap F^{\prime}$. Now $-d=$ $d S_{i}(j)^{2}=\left(\left(f \mid X_{F^{\prime}}\right)^{*} S_{i}(j)\right)^{2}=\left(d_{i j} S_{i}(j)^{\prime}\right)^{2}=-d_{i j}^{2}$, so $d_{i j}=\sqrt{d}>1$. Thus $h^{*} P_{i}=$ $\sqrt{d} P_{i}^{\prime}$ as 0 -cycles, with $P_{i}^{\prime}=h^{-1}\left(P_{i}\right)$, contradicting $\operatorname{deg}(h)=1$. This proves the claim and Lemma 3.2 .

Lemma 3.4. Let $X$ be a rationally connected projective threefold with only terminal singularities, and $\pi: X \rightarrow Y$ a conic bundle. Suppose that $f: X \rightarrow X$ is an endomorphism of degree $d>1$ and it descends to an endomorphism $h: Y \rightarrow Y$ of degree $e>1$. Then either $X$ is rational, or $d=e, X$ is non-Gorenstein, $f$ is étale over $X \backslash \operatorname{Sing} X$, and $K_{X}^{3}=0$.

Proof. By Lemma 2.2, $Y$ is a rational surface with at worst Du Val singularities. So $K_{Y}$ is Cartier and $K_{\widetilde{Y}}=\sigma^{*} K_{Y}$ if $\sigma: \widetilde{Y} \rightarrow Y$ is a minimal resolution.

By Proposition 2.7, we may assume that the (1-dimensional part) $D:=D_{1}(X / Y)$ $\neq \emptyset$. Replacing $f$ by some power, we may assume that $h^{-1}\left(D_{i}\right)=D_{i}$ for every irreducible component $D_{i}$ of $D$, as in Lemma 2.9. So $h^{*} D_{i}=d_{i} D_{i}$ for some $d_{i} \in \mathbb{N}$. Thus $K_{Y}+D=h^{*}\left(K_{Y}+D\right)+G$, where $G$ is an effective divisor having no common component with $D$. Inductively, for all $s>0$, we have

$$
K_{Y}+D=h^{*}\left(K_{Y}+D\right)+G=\left(h^{s}\right)^{*}\left(K_{Y}+D\right)+\sum_{i=0}^{s-1}\left(h^{i}\right)^{*} G .
$$

In view of Proposition 2.7, we may impose an extra assumption that $\sigma^{*} D . F^{\prime} \geq 4$ where $F^{\prime}$ is a general fibre of a $\mathbb{P}^{1}$-fibration on $\widetilde{Y}$ when $\widetilde{Y} \neq \mathbb{P}^{2}$ and $F^{\prime}$ is a line when $\tilde{Y}=\mathbb{P}^{2}$. We apply Lemma 2.6 and use the notation $X^{\prime} \rightarrow Y^{\prime}$ there. We 
may assume that $Y^{\prime} \rightarrow Y$ factors as $Y^{\prime} \rightarrow \widetilde{Y} \rightarrow Y$. Let $D^{\prime}:=D\left(X^{\prime} / Y^{\prime}\right)$ and let $\widetilde{D} \subset \widetilde{Y}$ be the image of $D^{\prime}$. By Lemma 2.5. $\left|K_{Y^{\prime}}+D^{\prime}\right| \neq \emptyset$, so $\left|K_{\widetilde{Y}}+\widetilde{D}\right| \neq \emptyset$ and $\left|K_{Y}+D\right| \neq \emptyset$.

Take an ample divisor $H$ on $Y$. Then for all $s>0$,

$$
H .\left(K_{Y}+D\right)=H .\left(h^{s}\right)^{*}\left(K_{Y}+D\right)+\sum_{i=0}^{s-1} H .\left(h^{i}\right)^{*} G \geq \sum_{i=0}^{s-1} H .\left(h^{i}\right)^{*} G .
$$

Thus $G=0$ and $K_{Y}+D=h^{*}\left(K_{Y}+D\right)$. If $K_{Y}+D \equiv 0$, then for the $F^{\prime}$ above we have $\sigma^{*} D \cdot F^{\prime}=\sigma^{*}\left(-K_{Y}\right) \cdot F^{\prime}=-K_{\widetilde{Y}} \cdot F^{\prime} \leq 3$, a contradiction to the extra assumption above. So $K_{Y}+D$ is not numerically trivial. By 18, Theorem 7.1.1; cf. also Remark 7.1.2, Theorem 2.6.5], there is a fibration $Y \rightarrow B \cong \mathbb{P}^{1}$ (for $g(B) \leq q(Y)=0$ ) with a general fibre $F$ such that

$$
K_{Y}+D \sim_{\mathbb{Q}} a F
$$

for some rational number $a>0$. Since $h^{*}\left(K_{Y}+D\right)=K_{Y}+D$, we have $h^{*} F \sim_{\mathbb{Q}} F$, so $h$ descends to an automorphism $h_{B}: B \rightarrow B$. Now $0=F \cdot a F=F \cdot\left(K_{Y}+D\right) \geq$ $F . K_{Y}$. Hence either $F \cong \mathbb{P}^{1}$ or $F$ is an elliptic curve. If $F \cong \mathbb{P}^{1}$, then for $F^{\prime}:=\sigma^{*} F$, we have $F^{\prime} \cdot \sigma^{*} D=F \cdot\left(-K_{Y}\right)=2$. This contradicts the extra assumption above.

We still have to consider the case where $F$ is elliptic and $Y \rightarrow B$ is an elliptic fibration.

Claim 3.5. (1) $h$ is étale over $Y \backslash \operatorname{Sing} Y$.

(2) Every fibre of the elliptic fibration $Y \rightarrow B$ is irreducible.

(3) We have $S(Y)=\emptyset$, so Sing $Y \neq \emptyset$.

(4) The Picard number $\rho(Y)=2$.

(5) If $\operatorname{deg}(f)=d=e=\operatorname{deg}(h)$, then $X$ is non-Gorenstein and $f$ is étale over $X \backslash$ Sing $X$, so $d K_{X}^{3}=\left(f^{*} K_{X}\right)^{3}=K_{X}^{3}$ and $K_{X}^{3}=0$.

We now prove Claim 3.5, Replacing $f$ by some power, we may assume that both $h$ and $h^{-1}$ stabilize every negative curve on $Y$ as in Lemma 2.10. (1) is proved in [18, Lemma 6.1.4].

(2) If $C$ is a curve in a reducible fibre, then $C$ is a negative curve and hence $h^{*} C=b C$ for some $b \in \mathbb{N}$, where $b^{2} C^{2}=\left(h^{*} C\right)^{2}=e C^{2}$ and $b=\sqrt{e}>1$. Thus $h_{B}^{*} P=b P$ for the point $P$ over which lies the curve $C$. This is impossible because $\operatorname{deg}\left(h_{B}\right)=1$.

Here is a note before continuing the proof of Claim 3.5. Note that $0=F \cdot a F=$ $F .\left(K_{Y}+D\right)=F . D$ and hence $D=\sum_{i=1}^{s} D_{i}$ with $D_{i}$ irreducible and the support of a fibre $F_{i}$. So $D \equiv w F$ for some $w>0$. Since $K_{\widetilde{Y}}^{2}=K_{Y}^{2}=(a F-D)^{2}=0$, the induced elliptic fibration $\widetilde{Y} \rightarrow \mathbb{P}^{1}$ (with a general fibre $\widetilde{F}$ say) is relatively minimal, and $\sigma: \widetilde{Y} \rightarrow Y$ is just the contraction of (-2)-curves in fibres of $\widetilde{Y} \rightarrow B$ into Du Val singularities.

(3) Note that $\widetilde{Y}=Y$ implies that there is a $(-1)$-curve on $Y$ (noting that $\widetilde{Y}$ is rational and $\left.K_{\widetilde{Y}}^{2}=0\right)$. So we have only to consider the case where there is a negative curve $E$ on $Y$. Then $h^{*} E=\sqrt{e} E$ as in (2). Also $E$ is horizontal to the elliptic fibration $Y \rightarrow B$ by (2), so $E . F>0$. Now $e(E . F)=h^{*} E \cdot h^{*} F=\sqrt{e} E . F$ and hence $e=\sqrt{e}$. This is impossible for $e=\operatorname{deg}(h)>1$ by the assumption. 
(4) Since $\operatorname{deg}(h)=e>1$ and $K_{\widetilde{Y}}=\sigma^{*} K_{Y}$ (and hence $K_{Y}$ ) are not pseudoeffective, $\rho(Y) \neq 2$ would imply that $h$ is polarized by some ample divisor $H$ so that $h^{*} H \sim \sqrt{e} H$ as in [18, Theorem 4.4.6]; this leads to $e(H . F)=h^{*} H . h^{*} F=\sqrt{e} H . F$, which is absurd.

(5) Note that $d / e=\operatorname{deg}(\ell \rightarrow f(\ell))$ for a general fibre $\ell$ of $\pi: X \rightarrow Y$. To distinguish, we write $f: X_{1}=X \rightarrow X_{2}=X, h: Y_{1}=Y \rightarrow Y_{2}=Y$ and $\pi_{i}: X_{i} \rightarrow Y_{i}$ (identical to $\pi$ ) so that $\pi_{2} \circ f=h \circ \pi_{1}$.

Suppose that $d=e$. We assert then that $X_{1}$ is isomorphic to the normalization $Z$ of $X_{2} \times_{Y_{2}} Y_{1}$. Indeed, $\pi_{1}: X_{1} \rightarrow Y_{1}$ and $f: X_{1} \rightarrow X_{2}$ factor as $X_{1} \rightarrow Z \rightarrow Y_{1}$ and $X_{1} \rightarrow Z \rightarrow X_{2}$, where $Z \rightarrow Y_{1}$ and $Z \rightarrow X_{2}$ are the natural projections. Since $\pi_{2}: X_{2} \rightarrow Y_{2}$ has connected fibres and $h$ is finite, $Z$ is irreducible. Note that the map $X_{1} \rightarrow Z$ is finite because so is $f: X_{1} \rightarrow X_{2}$. The map $X_{1} \rightarrow Z$ is also birational because $\operatorname{deg}\left(X_{1} / X_{2}\right)=\operatorname{deg}\left(Y_{1} / Y_{2}\right)=\operatorname{deg}\left(Z / X_{2}\right)$. Thus the map $X_{1} \rightarrow Z$ is an isomorphism and we can and will identify $X_{1}=Z$.

By (1), $f: X \rightarrow X$ is étale in codimension 1 , so we have $\left(^{*}\right): f$ is étale over $X \backslash \operatorname{Sing} X$ by the purity of the branch loci. Thus $X$ is non-Gorenstein. Indeed, a Gorenstein 3-dimensional terminal singularity is an isolated hypersurface singularity and hence has trivial local $\pi_{1}$ by a result of Milnor, so that $f^{s}: X \rightarrow X$ is étale of degree $d^{s}$ for any $s>0$ (by $(*)$ ), contradicting the fact that the rationally connected variety $X$ has finite $\pi_{1}$. This proves Claim 3.5.

We continue the proof of Lemma 3.4 Let $\rho$ be the spectral radius of $h^{*} \mid N^{1}(Y)$ and $L$ a non-zero $\mathbb{R}$-Cartier nef divisor such that $h^{*} L \equiv \rho L$. Note that $\rho \geq \sqrt{e}>1$ by Lemma 2.14. So $L$ is not parallel to $F$ and hence $L . F>0$ by the Hodge index theorem applied to the pullbacks on $\widetilde{Y}$. Thus $e(L . F)=h^{*} L . h^{*} F=\rho L . F$ and $\rho=e$. Since $e L^{2}=\left(h^{*} L\right)^{2}=(e L)^{2}$, we have $L^{2}=0$.

Claim 3.6. (1) We have $\overline{\mathrm{NE}}(Y)=\mathbb{R}_{\geq 0} L+\mathbb{R}_{\geq 0} F$ with $L$ and $F$ extremal rays.

(2) $L$ (replaced by its positive multiple) is an integral Cartier divisor with $h^{0}(Y, L) \geq 2$ and the Iitaka $D$-dimension $\kappa(Y, L)=1$.

(3) A multiple of $L$ is linearly equivalent to a general fibre of a $\mathbb{P}^{1}$-fibration $\psi: Y \rightarrow C \cong \mathbb{P}^{1}$.

We prove Claim 3.6

(1) Note that $S(Y)=\emptyset$ and $L^{2}=0=F^{2}$ imply that $L$ and $F$ are extremal and hence (1) follows because the Picard number $\rho(Y)=2$. Indeed, for $G=L$ or $F$, decompose $G \equiv G_{1}+G_{2}$ as a sum of pseudo-effective divisors. Let $G_{i} \equiv P_{i}+N_{i}$ be the Zariski decomposition. Since $S(Y)=\emptyset$, we have $N_{i}=0$ and hence $G_{i}=P_{i}$ is nef. Now $0=G^{2}=G_{1}^{2}+G_{2}^{2}+2 G_{1} \cdot G_{2}$ implies that $G_{i} \cdot G_{j}=0$. Then, by the Hodge index theorem, $G_{1}$ is parallel to $G_{2}$ and hence both $G_{i}$ are parallel to $G$. So both $L$ and $F$ are extremal.

(2) We can choose $F$ and an ample divisor $H$ to be generators of $N^{1}(Y)$. Replacing $L$ by its multiple, we may write $L=\varepsilon F+u H$ with $\varepsilon= \pm 1$ and $u \in \mathbb{R}$. Now $0=L^{2}=2 \varepsilon u F . H+u^{2} H^{2}$ implies that $u=(-2 \varepsilon F . H) / H^{2} \in \mathbb{Q}$. Thus we may assume that $L$ is integral and Cartier.

By the Riemann-Roch theorem and noting that L.F $>0$ and $\sigma^{*} K_{Y}=K_{\widetilde{Y}}=$ $-v \sigma^{*} F$ for some $v>0$ due to Kodaira's canonical divisor formula,

$$
h^{0}(Y, L)=h^{0}\left(\widetilde{Y}, \sigma^{*} L\right) \geq \frac{L\left(L-K_{Y}\right)}{2}+1>1 .
$$

So $\kappa(L)=1$ because $L^{2}=0$. 
(3) For the $L$ in (2), write $|L|=|M|+F i x$ with Fix the fixed part. Now $0=L^{2} \geq L . M \geq 0$ and $0=L^{2}=L . M+$ L.Fix $\geq M^{2}+$ L.Fix $\geq M^{2} \geq 0$. So $L . M=0$ and hence $L$ is parallel to $M$ by the Hodge index theorem. Also $M^{2}=0$ and hence $\mathrm{Bs}|M|=\emptyset$. Replacing $L$ by a multiple of $M$ we may assume that $L$ is a fibre of the fibration $\psi: Y \rightarrow C \cong \mathbb{P}^{1}$ derived from $\Phi_{|M|}$. Note that $K_{Y}=-v F$. Since $\sigma^{*} L . K_{\tilde{Y}}=L . K_{Y}=L .(-v F)<0$, our (3) is true. This proves Claim 3.6.

We continue the proof of Lemma 3.4. By Claim 3.6. (3), we may assume that $L$ is a general fibre of $\psi$. Since the arithmetic genus $p_{a}\left(D_{i}\right)=1$, we have $L . D>0$. Since $h^{*} L \equiv e L$, our $h: Y \rightarrow Y$ descends to an endomorphism $h_{C}: C \rightarrow C$ of degree $e>1$. By [4, Theorem 5.1] and replacing $f$ by some power, we may assume that $h_{C}(c)=c$ for a general point $c \in C$. Our $h$ induces an endomorphism $h \mid Y_{c}: Y_{c} \rightarrow Y_{c}$ of $Y_{c}:=\psi^{-1}(c)$, and this endomorphism is an automorphism for $\operatorname{deg}(h)=e=\operatorname{deg}\left(h_{C}\right)$. Let $X_{c}=\pi^{*} Y_{c}$. Since $Y_{c} \equiv L$ by our choice of $L$, we have $h^{*} Y_{c} \equiv e Y_{c}$. So $f: X \rightarrow X$ induces an endomorphism $f \mid X_{c}: X_{c} \rightarrow X_{c}$ of degree $d / e$.

By Claim 3.5, we may assume that $d>e$. Since $r:=Y_{c} . D=L . w F>0$, our $X_{c}$ is a smooth ruled surface with a ruling $X_{c} \rightarrow Y_{c} \cong \mathbb{P}^{1}$ and $r$ singular fibres $S_{i}(1)+S_{i}(2)$ (two intersecting (-1)-curves) lying over the $s$ points $P_{i} \in Y_{c} \cap D$. By Lemma 2.10 and relabelling, we have $\left(f \mid X_{c}\right)^{*} S_{i}(1)=\sqrt{d / e} S_{j}(1)$. Thus $\left(h \mid Y_{c}\right)^{*} P_{i}=\sqrt{d / e} P_{j}$ as 0 -cycles. This is impossible for $\operatorname{deg}\left(h \mid Y_{c}\right)=1$. Lemma 3.4 is proved.

Lemma 3.7. Let $X$ be a $\mathbb{Q}$-factorial rationally connected projective threefold with only terminal singularities, and $\pi: X \rightarrow Y$ an extremal contraction with $\operatorname{dim} Y=1$. Suppose that $f: X \rightarrow X$ is an endomorphism of degree $d>1$. Then either $X$ is rational; or $X$ is non-Gorenstein, $f$ is étale over $X \backslash \operatorname{Sing} X$, and $K_{X}^{3}=0$.

We now prove Lemma 3.7. Since the Picard number $\rho(X)=\rho(Y)+1=2$, we may assume that $f$ preserves each of the two extremal rays of $\overline{\mathrm{NE}}(X)$, so it descends to a surjective endomorphism $h: Y \rightarrow Y$ after replacing $f$ by its square.

Claim 3.8. Suppose that $h$ is an automorphism. Then $X$ is rational.

Proof. Choose a general $y=y_{0} \in Y$ such that $y_{i}:=h^{i}\left(y_{0}\right)$ is not in $D(X / Y)$ or $\pi(\operatorname{Sing} X)$ for all $i \geq 0$. Set $X_{i}:=\pi^{*}\left(y_{i}\right)$ which is a (smooth) del Pezzo surface. Then $f^{*} X_{i+1}=X_{i}$ and the restriction $f_{i}=f \mid X_{i}: X_{i} \rightarrow X_{i+1}$ is a finite surjective morphism of degree $d$. By Theorem 2.3. we may assume that $K_{X_{i}}^{2}=K_{X_{0}}^{2} \leq 4$ for all $i \geq 0$. Note that the Picard number $\rho\left(X_{i}\right)=\rho\left(X_{0}\right)$. We shall reach a contradiction later.

Let $N_{i}$ be the union of all negative curves (i.e., (-1)-curves) on $X_{i}$. By Lemma 2.10, both $f$ and $f^{-1}$ induce natural bijections (inverse to each other) between $N_{i}$ and $N_{i+1}$. Indeed, $f_{i}^{*} N_{i+1}(j)=\sqrt{d} N_{i}(j)$ with $\operatorname{deg}\left(f_{i}\right)=d$ if we label $N_{i}=$ $\sum_{j} N_{i}(j)$ such that $f_{i}^{-1} N_{i+1}(j)=N_{i}(j)$. Set $K_{i}=K_{X_{i}}$. Then $K_{i}+N_{i}=f_{i}^{*}\left(K_{i+1}+\right.$ $\left.N_{i+1}\right)+G_{i}$, where $G_{i}$ is an effective divisor having no common components with $N_{i}$. By iterating, for all $s>0$, we have:

$$
K_{0}+N_{0}=\left(f_{s} \circ f_{s-1} \circ \cdots \circ f_{0}\right)^{*}\left(K_{s+1}+N_{s+1}\right)+G_{0}+\sum_{i=1}^{s}\left(f_{i-1} \circ \cdots \circ f_{0}\right)^{*} G_{i} .
$$

Since $K_{i}^{2} \leq 4$, our $N_{i}$ is a loop $N_{i}^{\prime}$ plus a positive divisor $N_{i}^{\prime \prime}$, so both $K_{i}+N_{i}^{\prime}$ and $K_{i}+N_{i}$ are pseudo-effective (see [3, Lemma 2.3]); indeed, a del Pezzo surface of degree 6 contains a loop of $(-1)$-curves. Multiplying the above displayed equality by 
an ample divisor and letting $s \rightarrow \infty$, we see that $G_{i}=0$ for almost all $i$. Relabelling $X_{i}$, we may assume that $G_{i}=0$ for all $i \geq 0$. Thus, denoting $g_{s}:=f_{s} \circ f_{s-1} \circ \cdots \circ f_{0}$, we have

$$
\begin{aligned}
K_{0}+N_{0} & =g_{s}^{*}\left(K_{s+1}+N_{s+1}\right)=g_{s}^{*}\left(K_{s+1}+N_{s+1}^{\prime}\right)+g_{s}^{*} N_{s+1}^{\prime \prime} \\
& =g_{s}^{*}\left(K_{s+1}+N_{s+1}^{\prime}\right)+d^{(s+1) / 2} g_{s}^{-1} N_{s+1}^{\prime \prime} .
\end{aligned}
$$

Multiplying the equality by an ample divisor and letting $s \rightarrow \infty$, we get a contradiction.

By the above claim, we may assume that $\operatorname{deg}(h)>1$. Let $y_{0} \in Y$ be a general $h$-periodic point which is not contained in $\pi(\operatorname{Sing} X) \cup D(X / Y)$ or the branch locus of $h$ (see [4, Theorem 5.1]). We may assume that $h\left(y_{0}\right)=y_{0}$ after replacing $f$ by some power. Set $X_{0}:=\pi^{*}\left(y_{0}\right)$ which is a (smooth) del Pezzo surface. Then the restriction $f_{0}=f \mid X_{0}: X_{0} \rightarrow X_{0}$ is a finite morphism of degree $d_{0}:=d / \operatorname{deg}(h)$. If $d_{0}>1$, then $K_{X_{0}}^{2} \geq 6$ by [17] or [22, Theorem 3], so $X$ is rational by Theorem 2.3.

Therefore, we may assume that $d_{0}=1$ and $\operatorname{deg}(h)=\operatorname{deg}(f)=d$. So $f^{*} F \equiv d F$ for a general fibre $F$ of $\pi$. To distinguish, we write $f: X_{1}=X \rightarrow X_{2}=X$, $h: Y_{1}=Y \rightarrow Y_{2}=Y$ and $\pi_{i}: X_{i} \rightarrow Y_{i}$ (identical to $\pi$ ) so that $\pi_{2} \circ f=h \circ \pi_{1}$. As in the proof of Claim 3.5, $X_{1}$ is isomorphic to the normalization $Z$ of $X_{2} \times_{Y_{2}} Y_{1}$.

In particular, the ramification divisor $R_{f}$ is supported on fibres and we can write $R_{f} \equiv b F$, noting that every fibre is irreducible for $\rho(X / Y)=1$. If $b=0$, i.e. $R_{f}=0$, then $K_{X}^{3}=0$ and $X$ is non-Gorenstein, as in the proof of Claim 3.5. Thus we may assume that $b>0$.

Since the Picard number $\rho(X)=2$, we have $\operatorname{Nef}(X)=\mathbb{R}_{\geq 0} L_{1}+\mathbb{R}_{\geq 0} L_{2}$ with $L_{i}$ extremal rays. We assert that a general fibre $F$ of $\pi$ is an extremal ray in $\operatorname{Nef}(X)$. Indeed, if $F=G_{1}+G_{2}$ is the decomposition into nef divisors, then $0=\ell . F=\ell . G_{1}+\ell . G_{2}$ for a curve $\ell$ in $F$ and hence $\ell . G_{i}=0$, so $G_{i} \in \pi^{*} N^{1}(Y)$ and $G_{i}$ is parallel to $F$ (see [9, Lemma 3-2-5]). The assertion is proved. So we may assume that $L_{1}=F$ and hence $f^{*} L_{1} \equiv d L_{1}$. Also $L_{1}^{2}=F^{2}=0$ (for later use). We can also write $f^{*} L_{2} \equiv \lambda L_{2}$ for some $\lambda>0$.

Write $K_{X} \equiv a_{1} L_{1}+a_{2} L_{2}$. Since $-K_{X}\left|F \equiv-a_{2} L_{2}\right| F$ is ample we have $a_{2}<0$. Now

$$
K_{X}=f^{*} K_{X}+R_{f} \equiv\left(a_{1} d L_{1}+a_{2} \lambda L_{2}\right)+b L_{1} .
$$

Comparing coefficients of $L_{i}$, we get

$$
a_{1}=\frac{b}{1-d}<0, \quad \lambda=1 .
$$

So $-K_{X}$ is an interior point in $\operatorname{Nef}(X)$ and hence ample. Also $f^{*} \mid N^{1}(X)$ can be diagonalized as $\operatorname{diag}[d, 1]$ with respect to the basis $\left\{L_{1}, L_{2}\right\}$. Write $\overline{\mathrm{NE}}(X)=\mathbb{R}_{\geq 0} \ell_{1}+$ $\mathbb{R}_{\geq 0} \ell_{2} \subset N_{1}(X)$. Since $N_{1}(X)$ is dual to $N^{1}(X)$, we have $B=\operatorname{deg}(f)\left(A^{T}\right)^{-1}$ if $A$ (resp. $B$ ) is the matrix representation of $f^{*} \mid N^{1}(X)$ (resp. $\left.f^{*} \mid N_{1}(X)\right)$ with respect to some basis. Thus $f^{*} \ell_{i} \equiv r_{i} \ell_{i}$, where $\left\{r_{1}, r_{2}\right\}=\{1, d\}$. We may assume that $\ell_{1}$ is contained in $F$ so that $\pi$ is the contraction of the extremal ray $\ell_{1}$. Then $\ell_{1} \cdot L_{1}=0$, so $\ell_{1} \cdot L_{2}>0$. Thus $d\left(\ell_{1} \cdot L_{2}\right)=f^{*} \ell_{1} \cdot f^{*} L_{2}=r_{1} \ell_{1} \cdot L_{2}$, whence $r_{1}=d$. So $r_{2}=1$. Therefore, we have

$$
f^{*} L_{1} \equiv d L_{1}, \quad f^{*} L_{2} \equiv L_{2}, \quad f^{*} \ell_{1} \equiv d \ell_{1}, \quad f^{*} \ell_{2} \equiv \ell_{2} .
$$

$d\left(\ell_{2} \cdot L_{2}\right)=f^{*} \ell_{2} \cdot f^{*} L_{2}=\ell_{2} \cdot L_{2}$ implies that $\ell_{2} \cdot L_{2}=0$. Thus we have

$$
\ell_{i} \cdot L_{i}=0(i=1,2), \quad \ell_{i} . L_{j}>0(i \neq j) .
$$


Let $\pi_{2}: X \rightarrow Y_{2}$ be the contraction of the (necessarily $K_{X}$-negative) extremal ray generated by $\ell_{2}$. Then $f$ descends to an endomorphism $h_{2}: Y_{2} \rightarrow Y_{2}$ and the Picard number $\rho\left(Y_{2}\right)=\rho(X)-1=1$. Hence $\operatorname{dim} Y_{2} \neq 0$. If $\operatorname{dim} Y_{2}=2$, the result follows from Lemmas 3.2 and 3.4 .

Consider the case $\operatorname{dim} Y_{2}=1$. Since $\ell_{2} \cdot L_{2}=0, L_{2}$ is parallel to a fibre of $\pi_{2}$. Hence $L_{2}^{2}=0$. Take an ample $H$ and write $H=u_{1} L_{1}+u_{2} L_{2}$ with $u_{i}>0$. Then $H^{3}=0$ for $L_{i}^{2}=0$, which is absurd!

Consider the case $\operatorname{dim} Y_{2}=3$ so that $\pi_{2}$ is birational. Let $H$ be an ample generator of $\operatorname{Pic}\left(Y_{2}\right)$. Then $\pi_{2}^{*} H$ is extremal in $\operatorname{Nef}(X)$ as in the proof for the extremality of $L_{1}=F$ above and since $\rho\left(Y_{2}\right)=\rho(X)-1=1$. Hence we may assume that $L_{2}=\pi_{2}^{*} H$ because $\ell_{2} \cdot \pi_{2}^{*} H=0$ and $\ell_{2} \cdot L_{1}>0$ imply that $\pi_{2}^{*} H$ is not parallel to $L_{1}$. Set $h_{2}^{*} H=\alpha H$. Then $\alpha=1$ because $L_{2} \equiv f^{*} L_{2}=f^{*} \pi_{2}^{*} H=\pi_{2}^{*} h_{2}^{*} H=\alpha L_{2}$. Thus $\operatorname{deg}\left(h_{2}\right)=\left(h_{2}^{*} H\right)^{3} / H^{3}=1$, whence $\operatorname{deg}(f)=1$ for $h_{2}$ is the descent of $f$. This is a contradiction. This proves Lemma 3.7.

\section{Proofs of theorems AND COROLlaries}

In this section we shall prove Theorems 1.1, 1.2 and 1.3 and their corollaries below. In fact, Theorem 1.1 is a consequence of Lemmas 3.2 , 3.4 and 3.7 .

Corollary 4.1. Let $X$ be a $\mathbb{Q}$-factorial projective threefold with only terminal singularities and a surjective endomorphism $f: X \rightarrow X$ of degree $q^{3}>1$. Assume either one of the following three conditions:

(1) $X$ is Gorenstein.

(2) $K_{X}^{3} \neq 0$.

(3) The ramification divisor $R_{f}$ is non-zero.

Assume further that $(*): X$ is rationally connected and $-K_{X}$ is big. Then $X$ is rational, unless $\left(f^{s}\right)^{*} \mid N^{1}(X)=q^{s}$ id for some integer $s \geq 1$ (and hence $f$ is polarized).

The hypothesis $(*)$ in Corollary 4.1 above is satisfied if $(X, \Theta)$ is klt weak $\mathbb{Q}$-Fano for some $\Theta$ (cf. Lemma 2.11).

Corollary 4.2. With the situation as in Theorem $1.3(3)$, suppose that either $\operatorname{dim} Y=0$ and $X_{t}$ is smooth, or $\operatorname{dim} Y \in\{1,2\}$ and at least one $X_{i}(0 \leq i \leq t)$ satisfies either one of the following three conditions:

(1) $X_{i}$ is Gorenstein.

(2) $K_{X_{i}}^{3} \neq 0$.

(3) The ramification divisor $R_{f_{i}}$ is non-zero.

Suppose further that $X$ is rationally connected. Then $X$ is rational.

Remark 4.3. (1) In the situation of Theorem 1.3, the ramification divisor $R_{f_{k}}=$ 0 for some $k \in\{0,1, \ldots, t\}$, if and only if $R_{f_{i}}=0$ for all $i \in\{0,1, \ldots, t\}$, if and only if $f_{i}$ is étale over $X_{i} \backslash \operatorname{Sing} X_{i}$; see Lemma 2.18.

(2) By the proof of Claim 3.5 (5), in Theorem 1.1 and Corollaries 4.1 and 4.2 we have:

condition(1) $\Rightarrow$ condition(3); condition(2) $\Rightarrow$ condition(3). 
Remark 4.4. Some remarks on Theorem 1.3 related to the building blocks of endomorphisms:

(1) Every $X_{i}(0 \leq i \leq t)$ is $\mathbb{Q}$-factorial and has only terminal singularities and big $-K_{X_{i}}$; see [9] or [10, Proposition 3.37, Corollary 3.42], and Theorem $2.16(2)$.

(2) Note that $-K_{X}$ is big and $X$ is rationally connected, whenever $(X, \Theta)$ is klt weak $\mathbb{Q}$-Fano for some $\Theta$; see Lemma 2.11.

Now consider the case $\operatorname{dim} Y \in\{1,2\}$ in Theorem 1.3(3).

(3) Every general fibre of $X_{t} \rightarrow Y$ is $\mathbb{P}^{1}$ (when $\operatorname{dim} Y=2$ ) or a smooth Fano surface $=$ del Pezzo surface (when $\operatorname{dim} Y=1$ ), since $X_{t}$ has only terminal singularities.

(4) Suppose further that $X$ is rationally connected. If $\operatorname{deg}(h: Y \rightarrow Y) \geq 2$ and either the Picard number $\rho(Y) \neq 2$ or $f_{t}^{*} \mid N^{1}\left(X_{t}\right)=q$ id, then $h$ is polarized (cf. [18, Theorem 4.4.6] and [25, Lemma 2.2]), and hence the set of $h$-periodic points is dense in $Y$ by [4, Theorem 5.1], so there is a surjective endomorphism $h \mid X_{y}: X_{y} \rightarrow X_{y}$ for some general $y \in Y$, after $h$ is replaced by some power. Here $X_{y} \subset X$ is the fibre over $y$ and is $\mathbb{P}^{1}$ or del Pezzo.

The remark below gives sufficient conditions to descend $f$ to a surjective endomorphism $h: Y \rightarrow Y$ as in Theorems 1.1 and 1.3 .

Remark 4.5. Let $\pi: X \rightarrow Y$ be a $K_{X}$-negative extremal contraction and $f: X \rightarrow$ $X$ a surjective endomorphism. Then $f$, replaced by some power, descends to a surjective endomorphism $h: Y \rightarrow Y$ if any one of the following seven conditions is satisfied.

(1) $X$ is a klt weak $\mathbb{Q}$-Fano variety.

(2) $(X, \Theta)$ is a klt weak $\mathbb{Q}$-Fano variety for some $\Theta$.

(3) The cone $\overline{\mathrm{NE}}(X)$ has only finitely many extremal rays ( $K_{X}$ negative or non-negative).

(4) $\operatorname{dim} Y \leq 1$.

(5) The Picard number $\rho(X) \leq 2$.

(6) $X$ is a $\mathbb{Q}$-factorial projective threefold with only terminal singularities and big $-K_{X}$; the degree $\operatorname{deg}(f) \geq 2$.

(7) $\operatorname{dim} Y<\operatorname{dim} X$; see [25, Theorem 2.13, or Appendix].

Proof of Remark 4.5. The situation in Remark 4.5(6) is done by Theorem 2.16. If $\operatorname{dim} Y \leq 1$, then $\rho(X)=\rho(Y)+1 \leq 2$. If $\rho(X) \leq 2$, then $\overline{\mathrm{NE}}(X)$ has at most two extremal rays. Thus by Lemma 2.11 we have only to consider the situation in Remark 4.5(3). Our $f^{ \pm}$permutes extremal rays of $\overline{\mathrm{NE}}(X)$; see [25, Lemma 2.11]. Hence $f^{ \pm s}$ stabilizes every such extremal ray for some $s>1$. So Remark 4.5 is true (cf. [25, Lemma 3.6] for the flip case).

Proof of Theorem 1.2. Since $X$ is Fano, it is rationally connected and has only finitely many extremal rays $\mathbb{R}_{\geq 0}\left[C_{i}\right]$ all of which are $K_{X}$-negative; see Lemma 2.11] Let $X \rightarrow X_{1}$ be the composite of blowdowns between smooth threefolds such that $X_{1}$ is a primitive smooth Fano threefold in the sense of [15]. Replacing $f$ by some power, we may assume that $f$ descends to a surjective morphism $f_{1}: X_{1} \rightarrow X_{1}$. This is because every smooth $X^{\prime}$ appearing in between $X$ and $X_{1}$ is obtained by contracting a $K$-negative extremal ray, the cone $\overline{\mathrm{NE}}\left(X^{\prime}\right)$ is generated by finitely 
many extremal rays (the images of $\mathbb{R}_{\geq 0}\left[C_{i}\right]$ ) and a finite morphism permutes these rays; see [25, Lemma 2.11]. If $\rho\left(X_{1}\right)=1$, then $X_{1}$ is a smooth Fano threefold of Picard number one having an endomorphism $f_{1}: X_{1} \rightarrow X_{1}$ with $\operatorname{deg}\left(f_{1}\right)=$ $\operatorname{deg}(f)>1$, so $X_{1} \cong \mathbb{P}^{3}$ by [1] or [6], and we are done! If $\rho\left(X_{1}\right) \geq 2$, by [15, Theorem 5], $X_{1}$ has an extremal contraction of conic bundle type, so $X_{1}$ is rational by Theorem 1.1 This proves Theorem 1.2 .

Proof of Theorem 1.3. Let $X=X_{0} \cdots \rightarrow X_{1} \cdots \cdots \rightarrow X_{t}$ be a composition of $K_{X_{i}}$ flips and divisorial contractions so that there is no $K_{X_{t}}$-negative extremal contraction of birational type.

Claim 4.6. For every $0 \leq i \leq t$, the $X_{i}$ is $\mathbb{Q}$-factorial uniruled with only terminal singularities, $-K_{X_{i}}$ is big, $K_{X_{i}}$ is not nef, and there is a $K_{X_{i}}$-negative extremal contraction.

We prove Claim 4.6. The bigness of $-K_{X}$ implies that of $-K_{X_{i}}$; see Theorem 2.16. So we can write $-K_{X_{i}}=A_{i}+G_{i}$ with $A_{i}$ an ample $\mathbb{Q}$-divisor and $G_{i}$ an effective $\mathbb{Q}$-divisor. Thus $X_{i}$ is uniruled by [15, Theorem 1]. For the other parts, see [10, Proposition 3.37, Corollary 3.42, Theorem 3.7]. This proves Claim 4.6,

We return to the proof of Theorem 1.3. Let $X_{t} \rightarrow X_{t+1}=Y$ be a $K_{X_{t}}$-negative extremal contraction. Then $\operatorname{dim} Y \leq 2$ by the choice of $X_{t}$. Theorem 1.3 (1) and (2) follow from Theorem 2.16. For Theorem $1.3(3)$, if $\operatorname{dim} Y=0$, then $\rho\left(X_{t}\right)=1$ (so $f_{t}^{*} \mid N^{1}\left(X_{t}\right)$ is a scalar) and hence $-K_{X_{t}}$ is ample because $K_{X_{t}}$ is not nef. Thus (3) follows from Lemma 2.15. (4) is proved in Lemma 2.12. This proves Theorem 1.3 .

Proof of Corollary 4.2, Note that $\operatorname{deg}\left(f_{t}\right)=\operatorname{deg}(f)>1$. If $\operatorname{dim} Y=0$, by the assumption, $X_{t}$ is a smooth Fano threefold with a surjective endomorphism $f_{t}$ of degree $>1$, so $X_{t} \cong \mathbb{P}^{3}$ by [1] or [6], and we are done! Consider the case $\operatorname{dim} Y \in\{1,2\}$. By the proof of Theorem 1.1] (in three lemmas), we may assume that $R_{f_{t}}=0$. Then $R_{f_{i}}=0$ for all $i \in\{0,1, \ldots, t\}$ by Lemma 2.18, This proves Corollary 4.2 (cf. Remark 4.3).

Proof of Corollary 4.1, In the notation of Theorem 1.3 we may assume that $\operatorname{dim} Y$ $\in\{1,2\}$; see also the first paragraph in the proof of Lemma 2.15. Now Corollary 4.1 follows from Corollary 4.2 .

\section{ACKNOWLEDGEMENT}

The author would like to thank Hiromichi Takagi for suggesting Lemma 2.12(2), Shigefumi Mori for examples of conic bundles with isolated discriminant (cf. [16, (1.1.1)]), the audience of Kinosaki Symposium (October 2008) and Pacific Rim Mathematical Association Conference (July 2009) for the comments, and the referees for valuable suggestions which make the presentation better. This project is supported by an Academic Research Fund of NUS.

\section{REFERENCES}

[1] E. Amerik, M. Rovinsky and A. Van de Ven, A boundedness theorem for morphisms between threefolds. Ann. Inst. Fourier (Grenoble) 49 (2) (1999) 405-415. MR.1697369 (2000f:14056)

[2] G. Birkhoff, Linear transformations with invariant cones, Amer. Math. Monthly 74 (1967) 274-276. MR0214605(35:5454)

[3] J. A. Chen, M. Chen and D. -Q. Zhang, A non-vanishing theorem for $\mathbb{Q}$-divisors on surfaces, J. Algebra 293 (2) (2005) 363-384. MR2172344 (2006g:14014) 
[4] N. Fakhruddin, Questions on self maps of algebraic varieties, J. Ramanujan Math. Soc. 18 (2) (2003) 109-122. MR.1995861 (2004f:14038)

[5] C. Favre, Holomorphic self-maps of singular rational surfaces, Publ. Mat. 54 (2010), no. 2, 389432. MR 2675930

[6] J. -M. Hwang and N. Mok, Finite morphisms onto Fano manifolds of Picard number 1 which have rational curves with trivial normal bundles, J. Alg. Geom. 12 (2003) 627-651. MR.1993759 (2005f:14086)

[7] V. A. Iskovskikh, On the rationality problem for conic bundles, Duke Math. J. 54 (1987), no. 2, 271-294. MR.899398 (88i:14038)

[8] V. A. Iskovskikh, On the rationality problem for algebraic threefolds, Proc. Steklov Inst. Math. 218 (3) (1997) 186-227. MR1642381 (99g:14002)

[9] Y. Kawamata, K. Matsuda and K. Matsuki, Introduction to the minimal model problem, Algebraic geometry, Sendai, 1985 (T. Oda, ed.), Adv. Stud. Pure Math., 10, Kinokuniya and North-Holland, 1987, pp. 283-360. MR946243 (89e:14015)

[10] J. Kollár and S. Mori, Birational geometry of algebraic varieties, Cambridge Tracts in Math., 134, Cambridge Univ. Press, 1998. MR.1658959 (2000b:14018)

[11] J. Kollár and C. Xu, Fano Varieties with Large Degree Endomorphisms, arXiv:0901.1692.

[12] C. McMullen, Dynamics on $K 3$ surfaces: Salem numbers and Siegel disks, J. Reine Angew. Math. 545 (2002) 201-233. MR.1896103 (2003a:37057)

[13] M. Miyanishi, Algebraic methods in the theory of algebraic threefolds - surrounding the works of Iskovskikh, Mori and Sarkisov, Algebraic varieties and analytic varieties (Tokyo, 1981), pp. 69-99, Adv. Stud. Pure Math., Vol. 1, North-Holland, Amsterdam, 1983. MR.715647 (85b:14054)

[14] S. Mori, Threefolds whose canonical bundles are not numerically effective, Ann. of Math. (2) 116 (1982), no. 1, 133-176. MR662120(84e:14032)

[15] S. Mori and S. Mukai, Classification of Fano 3-folds with $B_{2} \geq 2$, Manuscripta Math. 36 (1981/82), no. 2, 147-162; Erratum, Manuscripta Math. 110 (2003), no. 3, 407. MR641971 (83f:14032) MR.1969009

[16] S. Mori and Y. Prokhorov, On $\mathbb{Q}$-conic bundles, Publ. Res. Inst. Math. Sci. 44 (2008), no. 2, 315-369. MR2426350 (2009e:14062)

[17] N. Nakayama, Ruled surfaces with non-trivial surjective endomorphisms, Kyushu J. Math. 56 (2002) 433-446. MR1934136 (2003j:14050)

[18] N. Nakayama, On complex normal projective surfaces admitting non-isomorphic surjective endomorphisms, Preprint 2 September 2008.

[19] N. Nakayama and D. -Q. Zhang, Building blocks of étale endomorphisms of complex projective manifolds, Proc. Lond. Math. Soc. (3) 99 (2009), no. 3, 725-756. MR2551469

[20] N. Nakayama and D. -Q. Zhang, Polarized endomorphisms of complex normal varieties, Math. Ann. 346 (2010), no. 4, 991-1018. MR2587100

[21] Yu. G. Prokhorov and V. V. Shokurov, Towards the second main theorem on complements, J. Algebraic Geom. 18 (2009) 151-199. MR2448282 (2009i:14007)

[22] D. -Q. Zhang, On endomorphisms of algebraic surfaces. Topology and geometry: commemorating SISTAG, pp. 249-263, Contemp. Math., Vol. 314, Amer. Math. Soc., Providence, RI 2002. MR1941637 (2004c:14070)

[23] D. -Q. Zhang, Automorphism groups and anti-pluricanonical curves, Math. Res. Lett. 15 (2008), no. 1, 163-183. MR2367182 (2009f:14072)

[24] D. -Q. Zhang, Dynamics of automorphisms of compact complex manifolds, Proceedings of The Fourth International Congress of Chinese Mathematicians (ICCM2007), Vol. II, pp. 678-689; also: arXiv:0801.0843.

[25] D. -Q. Zhang, Polarized endomorphisms of uniruled varieties, With an appendix by Y. Fujimoto and N. Nakayama, Compos. Math. 146 (2010), no. 1, 145-168. MR2581245

[26] Q. Zhang, Rational connectedness of log Q-Fano varieties, J. Reine Angew. Math. 590 (2006) 131-142. MR2208131 (2006m:14021)

Department of Mathematics, National University of Singapore, 10 Lower Kent Ridge Road, Singapore 119076, Singapore

E-mail address: matzdq@nus.edu.sg 